\title{
Uses and Attitudes of Old and Oldest Adults towards Self-Monitoring Health Systems
}

\author{
Ine D'Haeseleer \\ KU Leuven, e-Media Research Lab \\ Leuven, Belgium \\ ine.dhaeseleer@kuleuven.be \\ Dominique Schreurs \\ KU Leuven, ESAT-TELEMIC \\ Leuven, Belgium \\ dominique.schreurs@kuleuven.be
}

\author{
Kathrin Gerling \\ KU Leuven, e-Media Research Lab \\ Leuven, Belgium \\ kathrin.gerling@kuleuven.be \\ Christopher Buckingham \\ Aston University, Aston Triangle \\ Birmingham, United Kingdom \\ c.d.buckingham@aston.ac.uk
}

\author{
Bart Vanrumste \\ KU Leuven, e-Media Research Lab \\ Leuven, Belgium \\ bart.vanrumste@kuleuven.be \\ Vero Vanden Abeele \\ KU Leuven, e-Media Research Lab \\ Leuven, Belgium \\ vero.vandenabeele@kuleuven.be
}

\begin{abstract}
Oldest adults (80 years and over) are the fastest growing group in the total world population. This is putting pressure on national healthcare budgets, as the distribution of healthcare expenses is strongly age-dependent. One way of mitigating this burden may be to let older adults contribute to their own health directly by using self-management health systems (SMHS). SMHS might help older, including oldest, adults gain insight into their health status, and invite them to take action. However, while many studies report on user evaluations of older adults with one specific sensor system, fewer studies report on older adults' uses and attitudes towards integrated SMHS. Moreover, most studies include participants with mean ages of 65 rather than 80 . In this paper, we report on a qualitative study, consisting of a focus group interview and a user evaluation of an SMHS by 12 participants with a median age of 85 years. Three main findings were derived: Older adults (1) showed heterogeneity in computer skills, (2) found health technologies useful for others - not yet for themselves, and (3) perceived health technologies as a threat to social interaction. These findings suggest that health technologies are not ready for adoption by older adults yet, and further research on making them more accessible and desirable is required.
\end{abstract}

\section{CCS CONCEPTS}

- Human-centered Computing $\rightarrow$ Human computer interaction (HCI).

\section{KEYWORDS}

Older adults, self-management health systems, user evaluation

\section{ACM Reference Format:}

Ine D’Haeseleer, Kathrin Gerling, Bart Vanrumste, Dominique Schreurs, Christopher Buckingham, and Vero Vanden Abeele. 2019. Uses and Attitudes of Old and Oldest Adults towards Self-Monitoring Health Systems. In

Permission to make digital or hard copies of all or part of this work for personal or classroom use is granted without fee provided that copies are not made or distributed for profit or commercial advantage and that copies bear this notice and the full citation on the first page. Copyrights for components of this work owned by others than the author(s) must be honored. Abstracting with credit is permitted. To copy otherwise, or republish, to post on servers or to redistribute to lists, requires prior specific permission and/or a fee. Request permissions from permissions@acm.org.

Pervasive Health '19, May 20-23, 2019, Trento, Italy

(c) 2019 Copyright held by the owner/author(s). Publication rights licensed to ACM.
Pervasive Health '19: EAI International Conference on Pervasive Computing Technologies for Healthcare, May 20-23, 2019, Trento, Italy.

\section{INTRODUCTION}

The world's population is ageing. In Europe, the proportion of people aged $65+$ is projected to almost double from $16 \%$ to $29 \%$ between 2010 and 2060 [17], and the proportion of the oldest adults $(80+)$ is projected to almost triple [18]. This imposes increasing stress on healthcare budgets, as the distribution of healthcare costs is strongly age-dependent [1].

One solution to mitigate the pressure on healthcare provision may be to engage older adults to further partake in the creation and maintenance of their own health. Here, self-management health systems (SMHS) could be one solution: SMHS are integrated solutions that capture health-related data via sensors, wearables, and/or activity trackers in combination with surveys and self-reports, and communicate this health data back to the end-user via advanced data visualisations, possibly enriched with risk assessment and decision support. SMHS concord with a paradigm shift in healthcare from curative to preventive care, and from passive to active patients [9]. By allowing self-monitoring of well-being, detecting decline and applying timely interventions, SMHS are considered to have potential to enhance the lives of older adults, and extending the period of healthy independent ageing [16].

To date, many studies have investigated the uses and attitudes of older adults towards singular sensor systems (e.g., sleep monitors, activity trackers, blood pressure monitors). However, fewer studies have studied older adults' uses and attitudes towards integrated SMHS, e.g., [5, 12]. Most importantly, many of these studies include participants with mean ages around 65 rather than 80 [19]; studies on healthcare technology adoption and use by the oldest old are limited. Therefore, to better understand why and how SMHS can be weaved into the lives of older adults, we report on a qualitative study ${ }^{1}$ with 12 older adults (median age $=85$ ), investigating their uses and attitudes towards SMHS.

\footnotetext{
${ }^{1}$ This qualitative study is part of a larger research project, GRACE-AGE [14] that aims to design an SMHS to help older adults assess risks regarding well-being, mental health, and safety. In particular, GRACE-AGE aims to redesign a current risk assessment scale called the 'Galatean Risk and Safety Technology' (GRiST) [2] with an older user group in mind. We have to acknowledge that the GRiST version used for this study dates from 2016, which has been updated since.
} 


\section{RELATED WORK}

In recent years, we have witnessed the advent of trackers and sensor systems designed to offer novel ways of engaging older adults to self-manage their health. SMHS distinguish themselves from these by the fact that they present integrated solutions: they combine data from multiple sensors and/or self-reports (e.g., surveys, logbooks, pain scales). Additionally, this data is fed back to the end-user via advanced visualisations, possibly enhanced with risk assessments and decision support. Below, we discuss recent SMHS that have been evaluated by older adults'.

YourWellness is a tablet application, designed to support positive well-being management by older adults [7]. The system asks older adults to self-report on several parameters (e.g., mood, sleep and social interactions). On the basis of these self-reports, a quality score is generated for each of the parameter that defines the content of the message to provide tailored feedback. Doyle et al. [5, 6] evaluated YourWellness with seven older adults (age ranging from 64 to 76) who had lived in smart homes for four years using the application. The smart homes consisted of movement sensing, measuring energy use, and monitoring interactive physiological sensing (e.g., blood pressure and weight). The study showed that older adults welcomed feedback from sensor data to support self-management of wellbeing [5]. Interestingly, older adults did not find the SMHS obtrusive in their daily lives. Rather, they indicated that there was a positive change in their well-being due to using these technologies [5].

$O A S I S$ is a health monitoring system where older adults receive a personalised health profile, and can monitor themselves on the basis of data captured via six biomedical sensors. In addition, they can consult a virtual health coach designed to promote active ageing [13]. In an evaluation study with over hundred older adults $(55+)$ in four pilot sites across Europe, the system was tested [12] Results showed a promising future for SMHS. The majority of the participants (78\%) could complete all tasks during the testing phase. The usefulness for this SMHS was also reported high by $84 \%$ of the participants. The participants believed that this SMHS would improve their overall health and well-being.

The aforementioned studies included older adults $(65+)$ in their evaluation, but lacked inclusion of the oldest adults (80+). However, to the authors' knowledge, studies focusing on the oldest adults are rare. Here, we identified a pilot study by Conci et al. [3] that explored the attitudes of ten oldest adults (mean age $=81$ ) towards a tele-assistance service, augmented by a sensor network installed at home. This was studied by using a questionnaire at the beginning and at the end of the testing period, supplemented with regular visits to collect users' impressions. This study confirmed that oldest adults have difficulties when a new monitoring technology is introduced. They found that after using the system for three months, perceived usefulness, perceived safety, and pleasure and satisfaction decreased, contradicting some of the previous findings.

In conclusion, the aforementioned studies on older adults towards SMHS have somewhat inconclusive results. While YourWellness and OASIS $[5,12]$ report overall positive user evaluations, results of studies that include the oldest old, for example the study by Conci et al. [3], report more nuanced findings. To date, fewer studies focus specifically on the oldest adults. Therefore, this study investigates the uses and attitudes of older adults, including the oldest adults, towards SMHS and health technologies.

\section{METHOD}

To explore the uses and attitudes towards SMHS and health technologies among older adults, we conducted a focus group interview, including a user evaluation of one particular SMHS with 12 older adults.

\subsection{Participants}

Participants were enrolled via InnovAge [10], an organisation that facilitates care innovations. InnovAge invited older adults from their member list or from collaborating local services centres. Participants $(n=12)$ consisted of eight women and four men (median age 85$)$, living independently at home $(n=5)$ or in a service flat $(n=7)$, see Table 1.

Table 1: Demographic information on participants

\begin{tabular}{ccccl}
\hline code & gender & age & living area & comments \\
\hline P01 & female & 92 & flat & no computer experience \\
P02 & male & 91 & flat & no computer experience \\
P03 & female & 89 & home & no computer experience \\
P04 & male & 89 & home & \\
P05 & female & 89 & flat & no computer experience \\
P06 & female & 86 & flat & no computer experience \\
P07 & female & 84 & flat & \\
P08 & male & 83 & home & \\
P09 & female & 82 & flat & no computer experience \\
P10 & male & 74 & flat & \\
P11 & female & 69 & home & \\
P12 & female & 65 & home & \\
\hline
\end{tabular}

\subsection{Procedure}

The focus group interview took place in a local residential care centre and took approximately two and a half hours. First, participants were welcomed, the research team introduced the topic of SMHS. Afterwards, the team explained the reason for the study, and that halfway through the focus group participants would be invited to interact with GRiST, as an example of an SMHS designed to detect risks concerning mental health, safety and well-being [2]. The focus group interview itself was semi-structured and first addressed more general topics related to technologies or gadgets and participants' behaviour towards these technologies, to then zoom in on technologies specifically for managing health, like SMHS.

After about one hour, a hands-on user evaluation of GRiST was conducted in order to help participants gain insights in what an SMHS is, and in order to gain a better understanding of what exactly it could do for older adults. Participants were asked to fill out questionnaires related to their 'state of mind', 'health and care', and 'personality and way of thinking'. Afterwards they could receive a report on their risks. Figure 1 shows two screenshots of what the application looks like; an image about the overview with all topics, 
and an example of how questions are formulated. After this handson user evaluation, the focus group interview presented further questions to elicit participants' responses towards SMHS based on this salient experience.

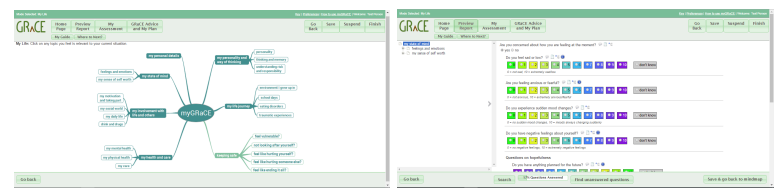

(a) Overview of all topics and (b) Questionnaire 'State of questionnaires Mind'

Figure 1: Screenshots GRiST software application

This user evaluation itself test took place in an adjacent room with notebooks installed and ready to start. Figure 2 gives an overview of the participants while performing the evaluation of the SMHS. Participants were asked to complete five basic tasks covering the most important goals and actions in the application: logging in with a specific username and password, filling out two different assessments, previewing their report, and checking their action plan. These tasks were written down on paper, so the participants could perform the tasks at their own pace.

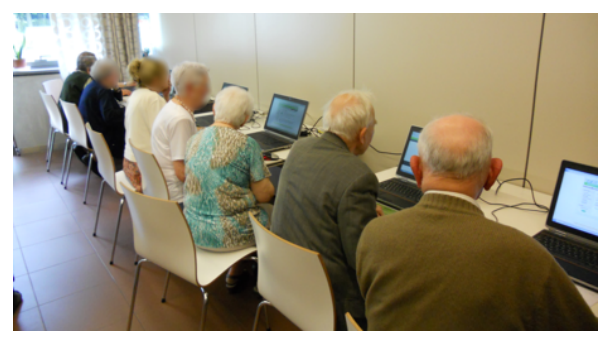

Figure 2: User evaluation of GRiST, one particular SMHS, with 12 participants

\subsection{Data Analysis}

During the focus group interview, audio and video materials were recorded. In addition, a picture-in-picture screen recorder [15] with video/audio of the webcam was used during the user evaluations. The recordings of the focus group interview were transcribed and entered in NVivo 11 [11] for analysis. The recordings of the individual user evaluations were viewed and analysed several times, including taking extended notes. Based on a qualitative approach, three themes were crafted, and discussed below.

\section{RESULTS}

Below we list the three main findings from the study: (1) heterogeneity in computer skills, (2) health technologies found useful for others - not yet for themselves, and (3) health technologies perceived as a threat to social interaction.

\subsection{Heterogeneity in computer skills}

The sample of 12 participants formed a heterogeneous group across ages and computer experience. Half of the participants reported to be familiar with working on a computer, notebook, or tablet. They had internet access at home and an email account which they checked regularly. One participant (P10) was volunteering as an IT technician, helping others with computing tasks. The other half of the participants mentioned they had no previous computer experience. For them, they reported, even completing basic computer tasks was challenging. We observed that the median age of computer experienced participants is 78.5 years, whereas the median age of those without experience is 89 .

Those participants who had never used a computer before were also anxious about the user evaluation. They expected not to be able to perform the tasks with the SMHS. Still, we encouraged them to sit down at a laptop and explore. Unfortunately, their expectations were confirmed. We noticed that learning how to handle a mouse was difficult, if not impossible for some participants during the short time of the study. During the user evaluation, these participants coped with nervous laughter, not being able to accomplish one task without receiving help.

$$
\begin{aligned}
& \text { P05: "I have used that (computer-) mouse... That was } \\
& \text { Greek to me." - aged } 89
\end{aligned}
$$

The other six participants who reported to be more computer experienced started the user evaluation test with a keen interest. However, P12 had to stop early as she feared getting a migraine attack. When analysing the recordings, we observed she was stuck during the first task already, always returning to the same (incorrect) page. Therefore, she was getting visibly frustrated. As she wanted to stay for the second part of the discussion, it might have been that she was looking for a 'graceful' exit.

P12: "I had lost the battle...I would have found it more interesting to fill out by using pen and paper. That would have progressed faster, and at least I would not have had a migraine attack." - aged 65

Finally, only five participants managed to complete all tasks using the SMHS. However, they as well reported the interaction to be difficult.

P07: "I thought it was very difficult. The interpretation of the questions, and what you had to do. First, you had to read all the questions and understand what they actually meant, and than you need to think about what is right for me... And afterwards, you still need to select the right buttons." - aged 84

\subsection{Health technologies found useful for others - not yet for themselves}

During the interview, participants all agreed on the importance of staying healthy when growing older. However, it was noteworthy that all participants who lived in a service flat $(n=7)$ had received an alarm bracelet. Strangely though, none of them actually wore this bracelet. Discussing this observation, they reported they did not feel they needed to wear it, as they were still doing fine.

moderator: "And are you wearing it at this moment?" P02: "No, we do not need this." - aged 91 
moderator: "Ok, so everyone has such an alarm, but nobody is wearing it?"

P07: "Yeah, that's true." - aged 84

Participants voiced the same pattern for other health trackers as well, these were typically stored somewhere in a drawer. We noticed a big difference between what participants uttered to be useful for others, and what they actually 'needed', and therefore wanted to use themselves. It was mentioned several times that they did not need this (e.g., monitoring device, health technology, or tool) yet. Later, when they would be in 'need', they might use these technologies.

P11: "Only when I would have any trouble, because in the end, I only bought this pedometer since I had problems with my hip, and I have no other problems, so I don't need all of this." - aged 69

\subsection{Health technologies perceived as a threat to social interaction}

Finally, we noted that almost all of the participants were volunteers. They helped out in the cafeteria, served as a technician, repaired clothes, entertained people, etc. Moreover, they all spend quite a lot of their time helping other people. They often still had quite busy schedules. This was found important as a manner to stay in touch with others.

However, most of the participants did not make use of social media, and even commented negatively on those who exposed there private life on social media.

$$
\begin{aligned}
& \text { P11: "I will never post something about me personally." } \\
& \text { - aged } 69 \\
& \text { P07: "Yeah, they made me take it [facebook account] in } \\
& \text { the (computer) lessons. (...) And apparently it is hard to } \\
& \text { get rid of it, otherwise I would have already deleted it." } \\
& \text { - aged } 84
\end{aligned}
$$

During the interview on SMHS, participants made a strong link to social media. They found that social media, and technology at large, alienates. It was reported several times that the social aspect of communicating face-to-face with others should remain paramount.

P02: "I would like to ask her (cf. author of this paper) to write somewhere in capitals: PEOPLE NEED TO LEARN TO COMMUNICATE BY USING LANGUAGE. Talk to each other, people walk past each other, but they don't say anything anymore." - aged 91

\section{DISCUSSION AND CONCLUSION}

This paper reports on the uses and attitudes of older adults towards SMHS and health technologies in general. A qualitative enquiry was conducted with 12 participants (median age 85), by means of a focus group interview. Three main findings were derived: (1) heterogeneity in computer skills, (2) health technologies found useful for others - not yet for themselves, and (3) health technologies perceived as a threat to social interaction.

Our findings can be interpreted with the technology acceptance model (TAM) in mind [4]. According to this model, adoption of a system can be predicted by two drivers: perceived usefulness and perceived ease-of-use. According to our study, perceived usefulness was low. The participants did not see the need to use health technologies themselves, as they were still in good health. While acknowledging the need for preventive care, which was deemed important for others but not for themselves, they mentioned several times that technologies helped reducing face-to-face communication with others. This growing phenomenon concerning social media, and technologies at large, was particularly disliked. Perceived ease-of-use was also found to be low. Only five out of 12 participants succeeded in performing basic tasks during the user evaluation. Half of the participants became anxious even before starting the user evaluation. Afterwards, the participants mentioned that they would have preferred filling out the questionnaires on paper. Therefore, informed by the TAM model, if SMHS are to be adopted by older and oldest adults, the following pitfalls need to be avoided: (1) SMHS should not be perceived as difficult to use, (2) SMHS should be found useful for preventive care, and (3) SMHS should not be perceived as a threat to face-to-face interactions.

Hence, participants in this study (median age 85) show a rather negative attitude towards health technologies, and a lack of intention to use them. These findings, although somewhat disheartening for developers of SMHS, confirm the results of Conci et al. [3]. These findings also confirm the results of Heart and Kalderon [8] who interviewed 123 older adults (mean age 80) on their willingness to adopt healthcare technologies, and found that the oldest adults expressed that computers were of no interest to them and that they did not feel a need to use them. Older adults were only willing to adopt modern healthcare technology, if the superior capacity to improve health status and quality of life - as compared with simpler traditional technologies - was obvious and irrefutable.

Further analysis is needed to investigate the attitudes towards SMHS, and how they should be designed for the oldest adults, to ensure use.

\section{LIMITATIONS}

For the recruitment of our participants we relied on a third party InnovAge [10]. While we succeeded in including the oldest adults, we acknowledge that there was self-selection in our recruitment process. Hence, it is likely that our sample predominantly included active older adults with an interest in technology. Additionally, since this is a study with a limited sample, and only one SMHS (dating from 2016), further research is needed to test with different participants and SMHS.

\section{ACKNOWLEDGMENTS}

We would like to thank InnovAge [10] for their participation and support. This research study was possible with the support of KIC EIT funding for GRACE-AGE.

\section{REFERENCES}

[1] Berhanu Alemayehu and Kenneth E Warner. 2004. The Lifetime Distribution of Health Care Costs. Health Services Research 39, 3 (June 2004), 627-642. https: //doi.org/10.1111/j.1475-6773.2004.00248.x

[2] Christopher D. Buckingham, Ann Adams, Laura Vail, Ashish Kumar, Abu Ahmed, Annie Whelan, and Eleni Karasouli. 2015. Integrating service user and practitioner expertise within a web-based system for collaborative mental-health risk and safety management. Patient Education and Counseling 98, 10 (Oct. 2015), 11891196. https://doi.org/10.1016/j.pec.2015.08.018 
[3] Mario Conci, Fabio Pianesi, and Massimo Zancanaro. 2010. Older Adults' Attitude Towards a Monitoring Technology. In Proceedings of the 28th Annual European Conference on Cognitive Ergonomics (ECCE '10). ACM, New York, NY, USA, 143146. https://doi.org/10.1145/1962300.1962328

[4] Fred D. Davis. 1985. A Technology Acceptance Model for Empirically Testing New End-user Information Systems: Theory and Results. Massachusetts Institute of Technology, Sloan School of Management, https://dspace.mit.edu/handle/1721.1/15192. Google-Books-ID: hbx8NwAACAAJ.

[5] J. Doyle, N. Caprani, and R. Bond. 2015. Older adults' attitudes to self-management of health and wellness through smart home data. In 20159 th International Conference on Pervasive Computing Technologies for Healthcare (PervasiveHealth) IEEE, https://ieeexplore.ieee.org/stamp/stamp.jsp?arnumber=7349388, 129-136. https://doi.org/10.4108/icst.pervasivehealth.2015.259279

[6] Julie Doyle, Andrea Kealy, John Loane, Lorcan Walsh, Brian O’Mullane, Carl Flynn, Andrew Macfarlane, Brennon Bortz, R Benjamin Knapp, and Rodd Bond. 2014. An integrated home-based self-management system to support the wellbeing of older adults. Fournal of ambient intelligence and smart environments 6,4 (2014), 359-383.

[7] Julie Doyle, Brian O’Mullane, Shauna McGee, and R. Benjamin Knapp. 2012 YourWellness: designing an application to support positive emotional wellbeing in older adults. In Proceedings of the 26th Annual BCS Interaction Specialist Group Conference on People and Computers. British Computer Society, http://dl.acm.org/citation.cfm?id=2377942, 221-226. http://dl.acm.org/citation. cfm?id=2377942

[8] Tsipi Heart and Efrat Kalderon. 2013. Older adults: Are they ready to adopt health-related ICT? International fournal of Medical Informatics 82, 11 (Nov. 2013), e209-e231. https://doi.org/10.1016/j.ijmedinf.2011.03.002

[9] C. Van Hoof. 2013. Addressing the healthcare cost dilemma by managing health instead of managing illness An opportunity for wireless wearable sensors. In 5th IEEE International Workshop on Advances in Sensors and Interfaces IWASI. IEEE, https://ieeexplore.ieee.org/document/6513758, 9-9. https://doi.org/10.1109/ IWASI.2013.6576064

[10] InnovAge. 2016. InnovAge. (2016). http://www.innovage.be/

[11] QSR International. 2015. NVivo 11. (2015).

[12] Viveca Jiménez-Mixco, Maria F. Cabrera-Umpiérrez, Maria T. Arrendondo, Maria Panou, Matthias Struck, and Silvio Bonfiglio. 2013. Feasibility of a Wireless Health Monitoring System for Prevention and Health Assessment of Elderly People. In 2013 35th Annual International Conference of the IEEE Engineering in Medicine and Biology Society (EMBC). IEEE, Osaka, Japan, 7306-7309.

[13] Viveca Jiménez-Mixco, Maria F. Cabrera-Umpiérrez, Alberto Esteban Blanco, Maria T. Arrendondo Waldmeyer, Daniel Tantinger, and Silvio Bonfiglio. 2012. Personalized e-Health for Elderly Self-care and Empowerment.pdf. AISC 153 (2012), 213-216.

[14] e-Media Research Lab. 2017. GRaCE-AGE | e-Media Lab. (2017). https://iiw. kuleuven.be/onderzoek/emedia/projects/grace-age/grace-age

[15] Blueberry Software Limited. 2016. FlashBack (BB FlashBack) try the best screen recorder. (2016). http://www.flashbackrecorder.com

[16] Christine Milligan, Celia Roberts, and Maggie Mort. 2011. Telecare and older people: Who cares where? Social Science \& Medicine 72, 3 (Feb. 2011), 347-354. https://doi.org/10.1016/j.socscimed.2010.08.014

[17] United Nations. 2015. World Population Prospects - Population Division - United Nations. (2015). http://esa.un.org/unpd/wpp/DataQuery/

[18] U.S. Department of State. 2007. Why population aging matters: A global perspective. (2007).

[19] Salifu Yusif, Jeffrey Soar, and Abdul Hafeez-Baig. 2016. Older people, assistive technologies, and the barriers to adoption: A systematic review. International journal of medical informatics 94 (2016), 112-116. https://doi.org/10.1016/j.ijmedinf. 2016.07.004 